\title{
Overexpression of PTGS2/COX-2 (Prostaglandin- Endoperoxidase Synthase-2) Gene Induces Angiogenesis, Metastasis and Invasion in TNBC (Triple-Negative Breast Cancer) In Vitro Cell Lines by Overexpressed Fzd-6 (Frizzled Class Receptor) Wnt/ Ca++ Signaling Pathway
}

\author{
Vikas Kumar Maurya* \\ Institute of Pharmacy, Bundelkhand University, India
}

\section{INTRODUCTION}

Cancer remains the scourge of mankind. After lung cancer, breast cancer is the second most common form of cancer. Breast cancer is the most common cancer in Indian women accounting for $27.7 \%$ with $1,62,468$ newly detected cases and 87,090 deaths in 2018. Triple-negative breast cancer (TNBC) prevalence in India varies between 27 to $35 \%$ of total breast cancer [1]. TNBC refers to a subgroup of breast cancer identified by the lack of ER (Estrogen Receptor), PR (Progesterone Receptor) \& HER (Human Epidermal Growth Factor Receptor). ER-/PR-/HER2- status is the main reason behind poor prognosis, failure of targeted therapy against these receptors like (Herceptin) and increased aggressiveness at an advanced stage [2]. Research paper and literature suggest that there is a lot of gene mutation, proteintruncating, receptor overexpression\& dysregulation like most common BRCA1/2 amplification, p53 \& Rbgene mutation, ER/ PR/HER2 overexpression, various signaling cascade inhibition, or overstimulation. Recent studies and research papers suggest that fzd-6 is particularly overexpressed in TNBC and some other cancer types like colorectal cancer and are the most studied receptors in TNBC which remains in the ON state forever [3].

FZD receptors are a group of subtypes(fzd1-10) from the GPCR family of receptors which signals through the Wnt ligand. Fzd signaling have 3 major pathways [4]

a) Wnt/beta catenin dependent(canonical) pathway b) Wnt/ca++ beta catenin independent pathway(noncannonical)

c) Wnt/pcp pathway

Wnt/ca++ payhway is still a less studied pathway but recent studies indicate that Like other various physiological pathways where calcium is involved as a secondary messenger and signal transducer when binding to calmodulin, here in wnt signaling it also activates various downstream proteins and promotes translocation of gene enhancers which then, in turn, induces expression of certain genes and finally protein synthesis. In the wnt/ca++ pathway studies showed that NFAT1 is the most notorious one which has been associated with enhancing COX-2 gene overexpression [5]; (Figure 1).

\section{EXPERIMENT}

In breast cancer, overexpression of COX-2 is associated with an indicator of poor prognoses, such as lymph node metastasis, poor differentiation, large tumor size [6]. A study demonstrated that the expression of NFAT1 \& its translocation to the nucleus promoted cancer cell invasion, while siRNA against NFAT1 successfully invasion ability of cancer cells. Moreover, they demonstrated that NFAT1 promoted cell invasion through the induction of COX2 in cancer cells and binds to the promoter region [7]. COX-2 is a critically important mediator of inflammation that significantly influences tumor angiogenesis, invasion, and metastasis. In a recent study overexpression of COX-2 is related to shaping the structure
Quick Response Code:

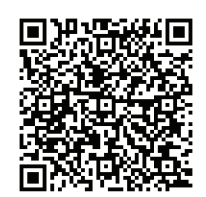

Address for correspondence: Vikas Kumar Maurya, Institute of Pharmacy, Bundelkhand University, India

Received: April 19, $2021 \quad$ Published: May 19, 2021

How to cite this article: Vikas KM. Overexpression of PTGS2/COX-2 (ProstaglandinEndoperoxidase Synthase-2) Gene Induces Angiogenesis, Metastasis and Invasion in TNBC (TripleNegative Breast Cancer) In Vitro Cell Lines by Overexpressed Fzd-6 (Frizzled Class Receptor) Wnt/Ca++ Signaling Pathway. 2021- 3(3) OAJBS.ID.000282. DOI: 10.38125/OAJBS.000282 
\& function of the extracellular matrix in primary and metastatic tumors and identified the potential role of COX-2in modifying the number of CAFs (Cancer- Associated Fibroblasts) that may have contributed to the altered extracellular matrix [8]. Based on these above findings and some pathways obtained from REACTOME we hypothesize the signaling pathway and downstream effects of calcium in signaling cascade as below (Figure 2). a

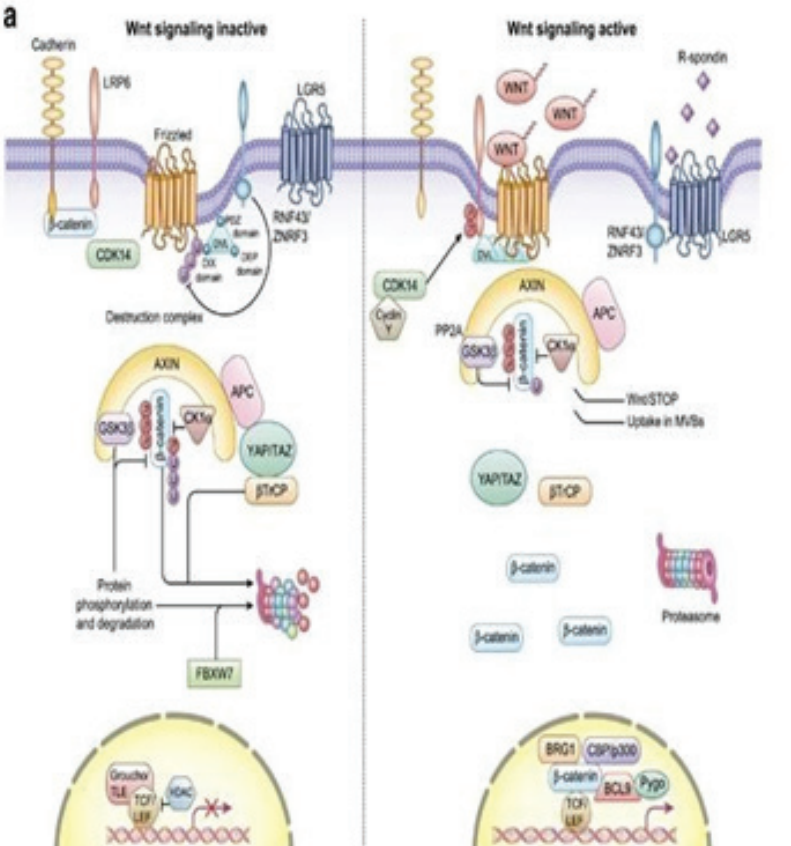

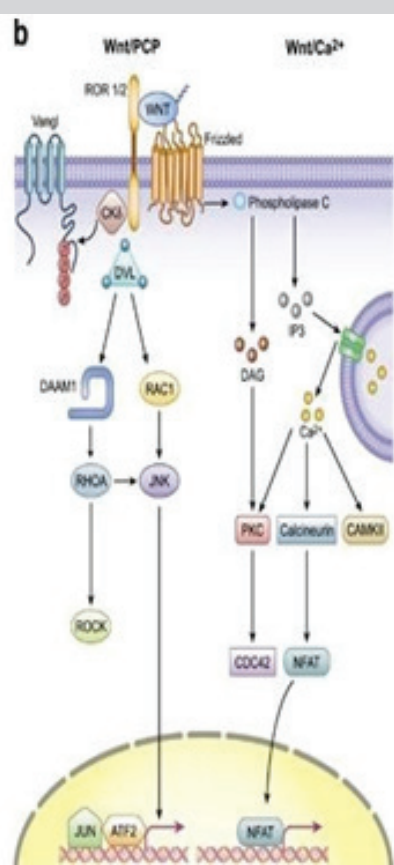

Figure 1: Wnt-fzd mediated signaling pathway.

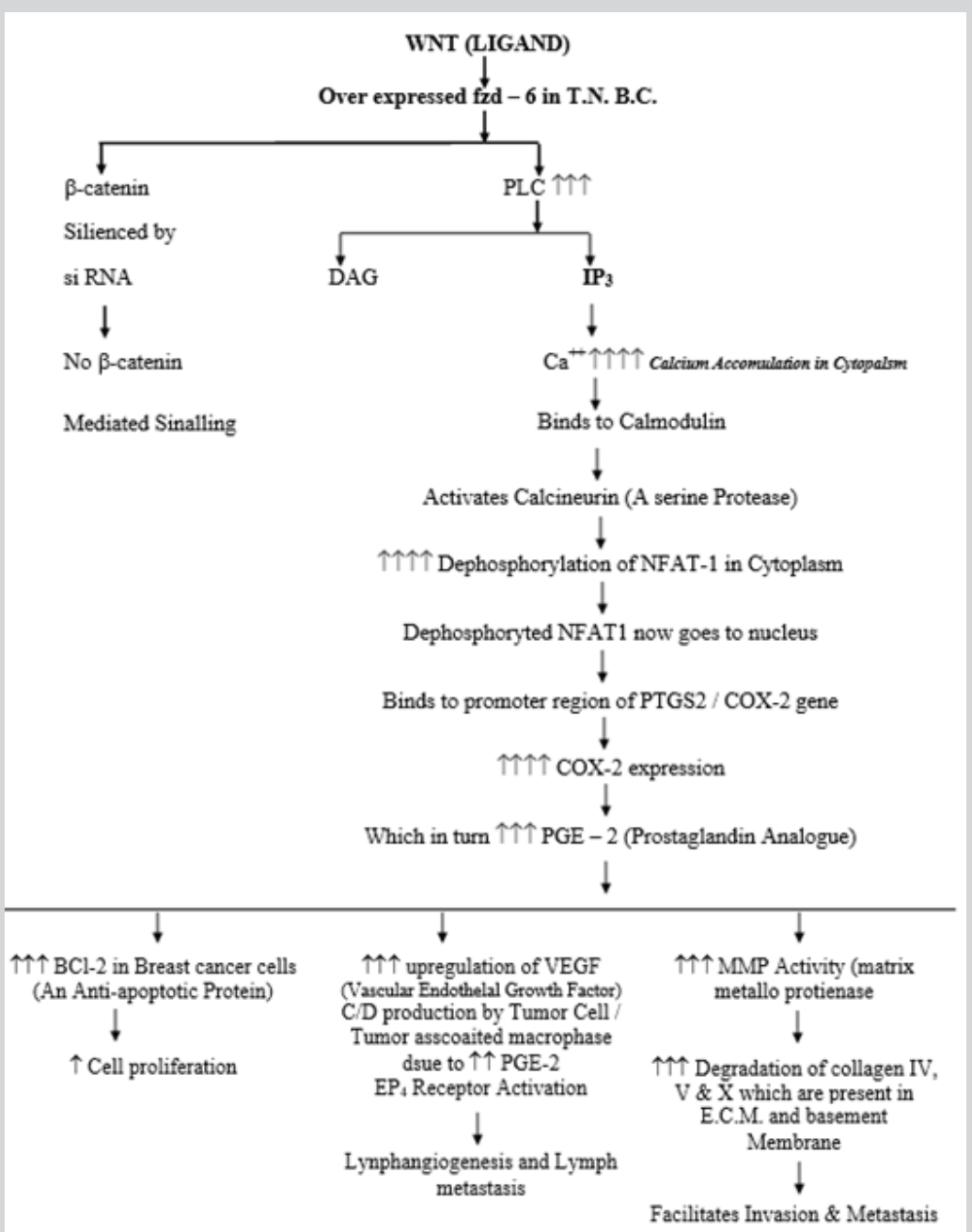

Figure 2. 
First, we will take two mammalian TNBC cell lines. Then we will silence the beta-catenin gene by siRNA (beta-catenin siRNA) to ensure that only the wnt/ca++ pathway is functioning. We will then leave both cell lines for 2 days of incubation to give a chance for ca++ accumulation in the cytoplasm and its binding with calmodulin to initiates a downstream signaling cascade. Now after incubation, we will treat one TNBC cell line with GSK3 BETA (Glycogen Synthase Kinase) and another cell line will be left untreated for another 1 day.

Now, after 1 day COX-2 mRNA will be isolated separately (by using oligo Dt beads) from both cell lines and will tag them with different fluorescent labels (say, GSK3Beta treated cell line with red \& untreated with green), mix them after tagging. Now we will perform microarray analysis to analyze mRNA expression levels. Due to the silencing of the beta-catenin pathway only the calcium/ wnt pathway will work in both cell lines along with the wnt/PCP pathway but our major focus is on the ca++ pathway.

GSK3 beta is a kinase which phosphorylates various protein and NFAT1. Treating one cell line with GSK3 beta will phosphorylate NFAT1 and prevent its nuclear localization which in turn will reduce expression of COX-2/PTGS2 gene whereas another cell line left untreated with GSK3 beta will still show nuclear NFAT1 localization and this will lead to overexpression of COX-2 gene. Now on the microarray profile, the cell line which was treated with GSK3 beta will show very little red fluorescence whereas the cell line without GSK3 beta treated will show very strong green fluorescence indicating that there is a high concentration of Cox-2 [9].

Expected outcomes- on observing both TNBC cell lines under electron microscope we will find, a cell line that has been left untreated with GSK3 beta has new blood vessels formed or dense capillary network, in extracellular matrix lots of collagen fiber will be present. On performing western blot for VEGF protein, we will find that there is a very high concentration of VEGF indicating the potential for angiogenesis. Another cell line that has been treated with GSK3 beta will have fewer capillaries, little to no new capillaries, very little VEGF concentration (western blot). Under a microscope with suitable stain, they will show clusters of degraded collagen fiber and to some degree type, 4 collagen degraded fibers of the endothelial basement membrane. This indicates that COX-2 overexpression is essential for metastasis [10].
These findings assist that COX-2 overexpression is essential for angiogenesis, invasion, metastasis, proliferation in TNBC cell lines. And in clinical practice CELECOXIB, a coX-2 inhibitor is administered in the early stage of TNBC have effects in slowing down cancer progression, which also supports that COX-2 has a very strong association with cancer. GSK3 beta biosimilar has the potential as a novel therapeutic agent which will keep NFAT1 phosphorylating and decreased expression of COX-2. Alternate strategy- we can also use different histopathological markers and stains for TNBC to compare the expression level of proteins or cellular structures in GSK3 beta treated and untreated cell lines [11].

\section{REFERENCES}

1. Jha PK, Ansari MA, Srivastava V, Verma AK, Mangla M (2020) Triplenegative breast cancer: Alarming burden and future challenges in Indian perspective. Journal of Scientific Research 65(2): 126-130.

2. Onitilo AA, Engel JM, Greenlee RT, Mukesh BN (2009) Breast cancer subtypes based on ER/PR and Her2 expression: Comparison of clinicopathologic features and survival. Clin Med Res 7(1-2): 4-13.

3. Pohl SG, Brook N, Agostino M, Arfuso F, Kumar AP, et al. (2017) Wnt signaling in triple-negative breast cancer. Oncogenesis 6(4): e310.

4. Huang HC, Klein PS (2004) The frizzled family: Receptors for multiple signal transduction pathways. Genome Biol 5(7): 234.

5. Malvina K, Veronica P, Maria FB (2020) The Wnt signalling pathway: A tailored target in cancer. Int J Mol Sci 21(20): 7697

6. Mosalpuria K, Hall C, Krishnamurthy S, Lodhi A, Hallman DM, et al. (2014) Cyclooxygenase-2 expression in non-metastatic triple-negative breast cancer patients. Mol Clin Oncol 2(5): 845-850.

7. Wang L, Wang Z, Li J, Zhang W, Ren F, et al. (2015) NFATc1 activation promotes the invasion of U251 human glioblastoma multiforme cells through COX-2. Int J Mol Med 35(5): 1333-1340.

8. Krishnamachary B, Stasinopoulos I, Kakkad S, Penet MF, Jacob D, et al. (2017) Breast cancer cell cyclooxygenase-2 expression alters extracellular matrix structure and function and numbers of cancer associated fibroblasts. Oncotarget 8(11): 17981-17994.

9. Vijay GV, Zhao N, Den Hollander P, Toneff MJ, Joseph R, et al. (2019) $\mathrm{GSK}_{3} \beta$ regulates epithelial-mesenchymal transition and cancer stem cell properties in triple-negative breast cancer. Breast Cancer Res 21(1): 37.

10. https://reactome.org/PathwayBrowser/

11. Zhan T, Rindtorff N, Boutros M (2017) Wnt signaling in cancer. Oncogene 36(11): 1461-1473. 